\title{
DESCRIÇÃO DO COMPORTAMENTO DA FORÇA DO KITE EM RELAÇÃO AO SEU POSICIONAMENTO E AO DESLOCAMENTO DA BARRA DE CONTROLE
}

\author{
GRAD. VICTOR WIGNER TREMEA \\ Graduado em Educação Física pela Universidade Federal do Rio Grande do Sul (ESEF/UFRGS) \\ (Porto Alegre - Rio Grande do Sul - Brasil) \\ e-mail:vwtremea@gmail.com
}

MS. LARA ELENA GOMES

Doutoranda em Ciências do Movimento Humano pela Universidade Federal do Rio Grande do Sul (ESEF/UFRGS), Professora do Colegiado de Educação Física da Universidade Federal do Vale do São Francisco (UNIVASF) (Petrolina - Pernambuco - Brasil)

e-mail: lara.gomes@univasf.edu.br

\section{DR. JEFFERSON FAGUNDES LOSS}

Doutor em Engenharia pela Universidade Federal do Rio Grande do Sul (UFRGS),

Professor do Departamento de Educação Física (ESEF/UFRGS)

(Porto Alegre - Rio Grande do Sul - Brasil)

e-mail: jefferson.loss@ufrgs.br

\section{RESUMO}

Introdução. No kitesurfing, os velejadores deslocam-se alterando o ângulo de ataque do kite de duas formas: mudando o kite na janela de vento e movimentando a barra de controle. Objetivo. Descrever o comportamento da força do kite em relação ao seu posicionamento e à distância percorrida pela barra de controle. Métodos. Foram utilizados sistemas de videogrametria e dinamometria. Duas situações foram testadas: movimentando o kite na janela de vento e deslocando a barra. Resultados. A força aumentou quando a barra foi abaixada. Movimentando o kite, a zona de força foi definida pela combinação de ângulos verticais menores que $60^{\circ} \mathrm{com}$ ângulos horizontais menores que $20^{\circ}$. Conclusão. A força aumenta quando a barra é abaixada. O ângulo vertical pode exercer maior influência na força.

PALAVRAS-CHAVE: Esportes radicais; esportes náuticos; janela de vento; ângulo de ataque. 


\section{INTRODUÇÃO}

O kitesurfing é um esporte náutico relativamente novo, que vem crescendo rapidamente em popularidade (NICKEL et al., 2004; SPANJERSBERG; SCHIPPER, 2007). Segundo Costa (2006), esse esporte promove uma relação intensa com a natureza, podendo ser praticado em todos ambientes aquáticos, onde exista a incidência de vento sem a obstrução de morros ou outros obstáculos.

Durante a sua prática, o indivíduo - por meio de um kite (pipa) controlável - utiliza a energia do vento para deslizar com uma prancha sobre a água (BLOUIN JUNIOR; ISABELLA; RODDEN, 2007; VERCRUYSSEN et al., 2009). Esse deslocamento é resultado da interação entre forças geradas em dois meios fluidos: a água (em relação à prancha) e o ar (em relação ao kite).

O kite é uma estrutura em forma de um fólio, com armação inflável e com área que varia de 5 a $20 \mathrm{~m}^{2}$, sendo que os mais utilizados são aqueles com área entre 8 e 16 m² (BLOUIN JUNIOR; ISABELLA; RODDEN, 2007; VERCRUYSSEN et al., 2009). Os kites mais novos são presos ao praticante por quatro linhas de aproximadamente $25 \mathrm{~m}$. Duas linhas se ligam ao bordo de ataque e, próximo ao praticante, se unem, passando dentro da barra de controle e terminando no chicken loop, o qual é uma estrutura em forma de argola (BLOUIN JUNIOR; ISABELLA; RODDEN, 2007). O praticante prende o chicken loop ao trapézio (dispositivo com um gancho que é preso ao redor da cintura) ou à cadeirinha (dispositivo com um gancho e com alças para as pernas que envolve o quadril). As outras duas linhas saem do bordo de fuga e prendem-se cada uma em uma das extremidades da barra de controle (BLOUIN JUNIOR; ISABELLA; RODDEN, 2007).

O praticante controla o kite manipulando a barra de controle, sendo que a região em que o kite voa em relação ao indivíduo é denominada janela de vento (Figura I). Esta, por sua vez, é representada por um quarto de uma esfera (BLOUIN JUNIOR; ISABELLA; RODDEN, 2007). A periferia dessa região é denominada zona neutra - área em que o kite apresenta menores valores de força (LUNDGREN et al., 2007) - e a região mais central é conhecida como zona de força - área que o kite tem maiores valores de força (BLOUIN JUNIOR; ISABELLA; RODDEN, 2007).

Na prática do kitesurfing, para desloca-se e para realizar manobras, o indivíduo necessita alterar o ângulo de ataque do kite. Isso é realizado por meio de duas maneiras: (I) movimentando o kite da zona neutra para a zona de força da janela de vento, e (2) movimentando a barra de controle para cima e para baixo. Os velejadores utilizam combinações dessas duas formas, pois ambas alteram a posição do kite em relação ao vento e em relação ao praticante, e consequentemente, modificam a força que o kite produz. 


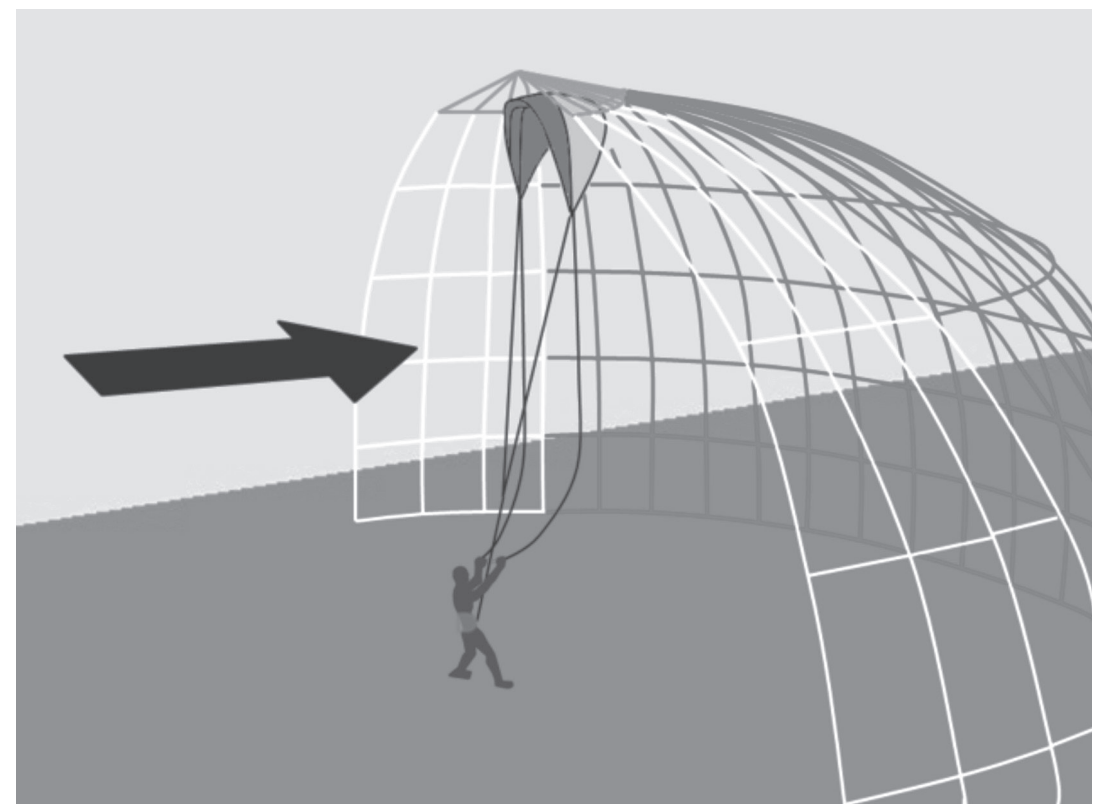

Figura I. A janela de vento está representada pelas linhas que formam um quarto de esfera. As linhas brancas e as de cor cinza claro ilustram a zona neutra, enquanto que as linhas de cor cinza escuro representam a zona de força da janela de vento. Já a seta preta representa a direção do vento.

No entanto, apesar de ser conhecido o funcionamento da janela de vento, não foram encontrados estudos que tenham mensurado os valores de força do kite dentro da janela de vento. Ainda, sabe-se que abaixando a barra de controle, os valores de força do kite aumentam, porém somente foi encontrado um relato na internet de um estudo que avaliou a força durante a mudança da posição da barra (PAELINCK, 20 I0). Logo, surge a seguinte questão: como é o comportamento da força exercida pelo kite em relação ao seu posicionamento na janela de vento e em relação à distância percorrida pela barra de controle?

Como os equipamentos apresentaram diversas mudanças importantes na recente história do kitesurfing, e para continuar esse processo evolutivo que torna o esporte mais seguro e melhora o desempenho, são necessários mais dados científicos (LUNDGREN et al., 2007). Não obstante, a produção científica sobre o kitesurfing é pouca e se concentra, até o momento, em analisar as lesões relativas a esse esporte (LUNDGREN et al., 2007) e os aspectos fisiológicos (VERCRUYSSEN et al.,2009). Sendo assim, a análise de movimento e das forças externas atuantes sobre o praticante pode vir a colaborar na evolução técnica do esporte e 
no desenvolvimento de equipamentos, visto que pouco se sabe sobre o estresse mecânico e como o sistema músculo esquelético é afetado. Além disso, esse tipo de análise compreende o primeiro passo para uma análise posterior das forças internas do corpo do velejador, o que terá consequências na prevenção de lesões e na preparação física.

Com base nessas informações, esse trabalho teve como objetivo: descrever o comportamento da força exercida pelo kite em relação ao seu posicionamento na janela de vento e em relação à distância percorrida pela barra de controle. Ainda, baseado nos conhecimentos existentes sobre o comportamento das forças dinâmicas com a alteração do ângulo de ataque de objetos com forma de fólio (SANDERS, 1999), na descrição do comportamento da força do kite ao subir e descer a barra de controle (BLOUIN JUNIOR; ISABELLA; RODDEN, 2007), e no relato de Paelinck (20I0) sobre o comportamento da força do kite em relação ao ângulo de ataque, é possível estabelecer as seguintes hipóteses: (I) os valores de força aumentam à medida que a barra de controle é abaixada, devido ao aumento o ângulo de ataque do kite; e (2) os valores de força aumentam à medida que o kite se posiciona na zona de força da janela de vento, visto que o ângulo de ataque aumenta dessa forma.

\section{MATERIAIS E MÉTODOS}

\section{AQUISIÇÃO DE DADOS}

A coleta dos dados ocorreu em Tramandaí - Rio Grande do Sul, em um dia com poucas nuvens, e vento sul (paralelo à praia) com velocidade variando entre $9,8 \mathrm{~m} / \mathrm{s}$ e I I,3 m/s. A velocidade do vento foi verificada a cada cinco minutos durante a coleta de dados com um anemômetro (modelo Skywatch-xplorer I da marca JDC).

Foi utilizado um Kite (modelo YARGA HYBRID SLE 2007; de área 9,5 m²; tipo LED; da marca BEST). Dois marcadores reflexivos foram posicionados na linha formada pela união das linhas que saem do bordo de ataque do kite com uma distância entre eles de $20 \mathrm{~cm}$ e mais dois marcadores foram posicionados nas extremidades da barra de controle (Figura 2).

Duas câmeras de vídeo digital (VPC-WHI - Sanyo) foram usadas para capturar o deslocamento de marcadores reflexivos com uma taxa de amostragem de 30 $\mathrm{Hz}$ e resolução espacial de 640×480 pixels. Foi usado um calibrador (modelo 5.3 da marca Peak Performance), o qual definiu um volume. $\bigcirc$ centro desse volume foi posicionado a 3,00 m de uma câmera e a 3,64 m da outra câmera, sendo o espaço entre as câmeras de 3,52 m. 
Para registrar a força do kite, que é uma força de tração, o chicken loop foi preso por meio de um mosquetão em uma extremidade da célula de carga (modelo ZX 250 Alfa Instrumentos - SP - com sensibilidade de $2 \mathrm{mVN}$ e capacidade para $2500 \mathrm{~N}$ ), a qual mede forças de tração ou compressão, assim, essa célula de carga pode medir força em uma única direção, mensurando - no presente estudo - somente a força resultante exercida pelo kite. Enquanto que, com auxilio de mosquetões, a outra extremidade da célula de carga foi presa a uma mala cheia de areia e ao gancho de reboque de um carro (Figura 3). Essa célula de carga estava ligada a um equipamento Miotec (Miotec equipamentos Biomédicos Ltda), o qual estava conectado a um computador (modelo PCG7 I 54L da marca Sony Vaio). A conexão com o reboque do carro foi feita com intuito de não permitir que a célula de carga se afastasse do carro a ponto de romper o fio que a conectava ao equipamento Miotec. A taxa de amostragem da dinamometria foi de $2000 \mathrm{~Hz}$ e o software utilizado para registrar os valores de força foi o Miograph.

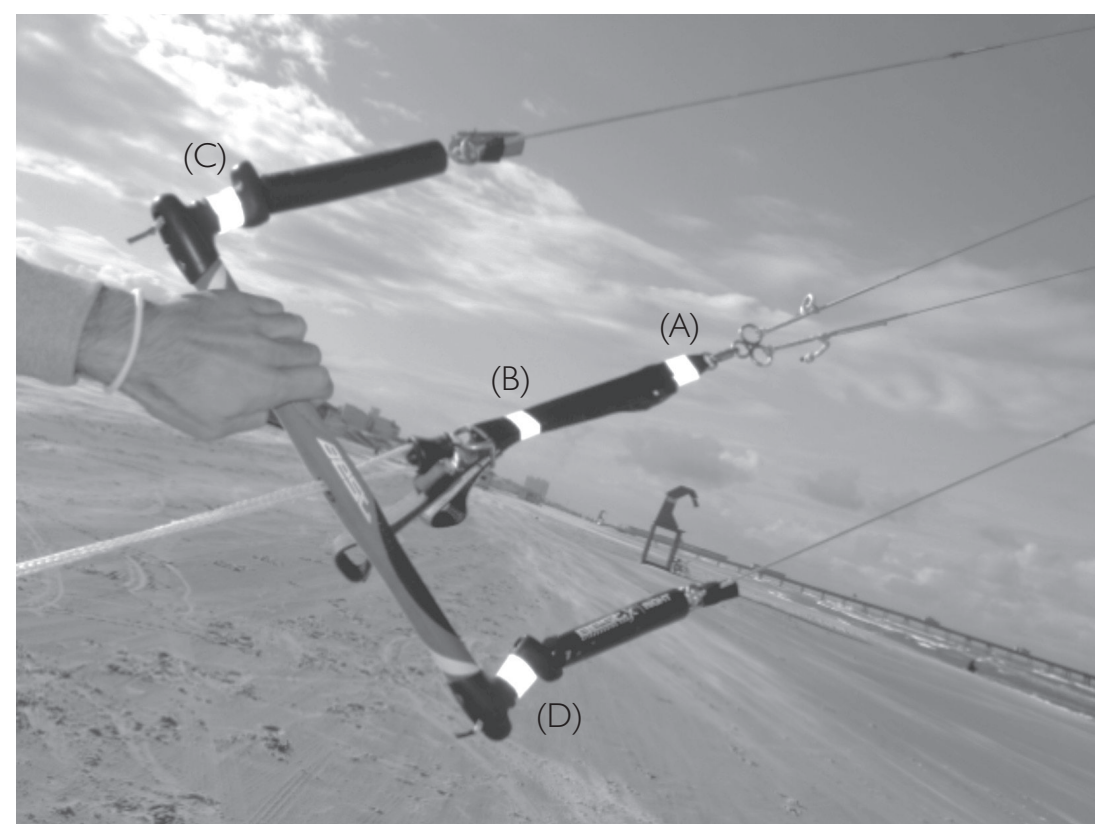

Figura 2. Marcadores reflexivos. (A) e (B) marcadores posicionados na linha formada pela união das linhas que saem do bordo de ataque do kite. (C) e (D) marcadores posicionados nas extremidades da barra de controle. 
Como a coleta envolveu a aquisição de dados cinemáticos e cinéticos de forma independente, foi necessário utilizar um sistema eletrônico de sincronização, o qual quando acionado forneceu, simultaneamente, um sinal elétrico - observado no software Miograph - e um sinal luminoso - observado em todas as câmeras - possibilitando a sincronização entre esses dois sistemas de medida (LOSS et al., 1997). Além disso, o sinal luminoso também foi usado como forma de sincronizar as câmeras.

Foram realizados dois diferentes testes: ( I ) duas repetições de abaixar e levantar a barra de controle (Figura 4A e 4B), com o kite na posição mais elevada da zona neutra da janela de vento (posição correspondente às linhas verdes da Figura I). E (2) cinco movimentos do kite com forma de oito na janela de vento, sendo que cada oito compreende dois mergulhos do kite na janela de vento, ou seja duas passagens do kite na zona de força (Figura 5). Para a realização desses testes, um praticante de kitesurfing experiente se posicionou de pé, atrás da mala cheia de areia com o intuito de manipular a barra de controle, executando os movimentos analisados em cada teste. Os comandos necessários para execução dos movimentos dos testes do presente estudo são comuns na prática do esporte, por isso, foram realizados por um praticante de kitesurfing experiente (três anos de prática).

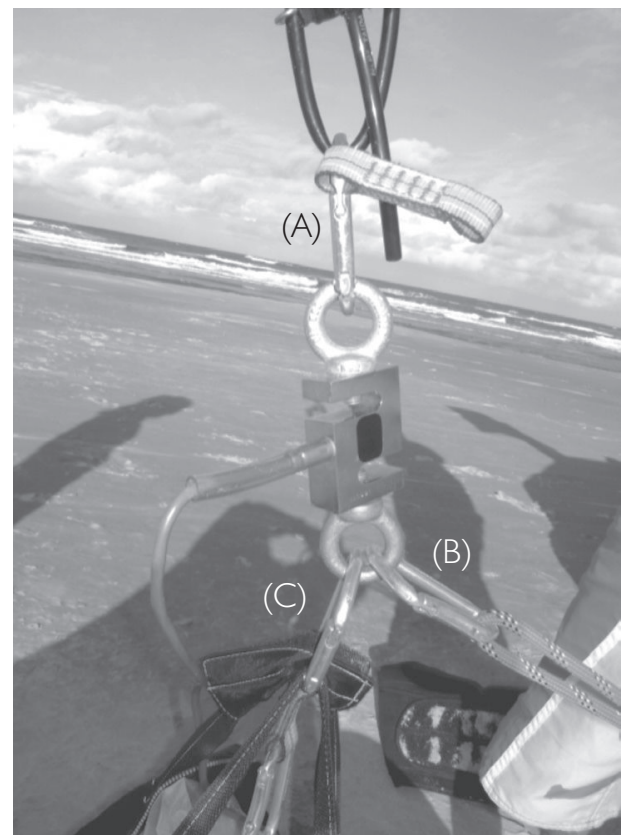

Figura 3. Célula de carga. (A) Mosquetão preso a célula de carga e ao chicken loop do kite. (B) Mosquetão preso à célula de carga e a um carro. (C) Mosquetão conectado à célula de carga e a uma mala cheia de areia. 
presente estudo faz parte de um trabalho maior, o qual foi aprovado pelo Comitê de Ética da universidade (protocolo número 2008249).

\section{ANÁLISE DOS DADOS}

Os marcadores reflexivos foram digitalizados manualmente no software Digital Video for Windows (BARROS et al., 1999). As coordenadas tridimensionais desses pontos foram obtidas por meio do método de transformação linear direta no mesmo software. Todos os outros procedimentos para análise dos dados foram realizados no software Matlab (versão 7.5).

As coordenadas tridimensionais foram filtradas por meio de um filtro passa baixa de ordem três, e a frequência de corte foi determinada por meio da Análise Residual proposta por Winter (2005). As posições filtradas foram interpoladas para que a taxa de amostragem da cinemetria coincidisse com a da dinamometria.

(A)

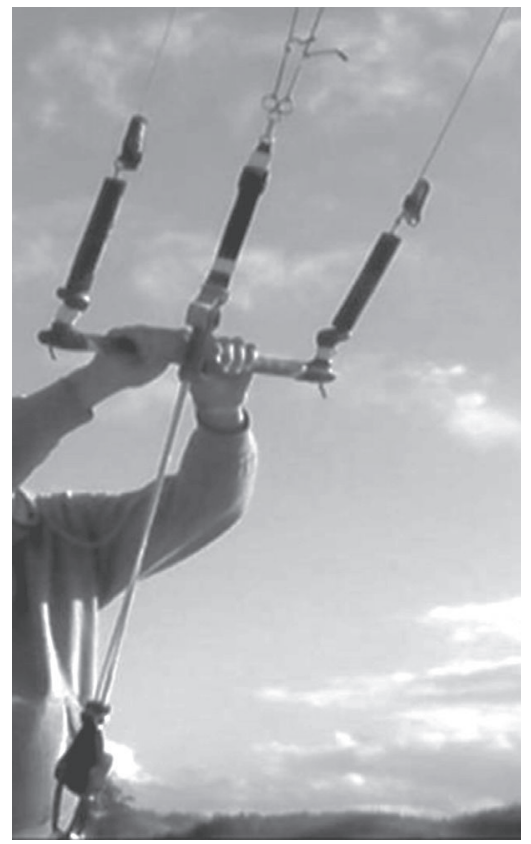

(B)

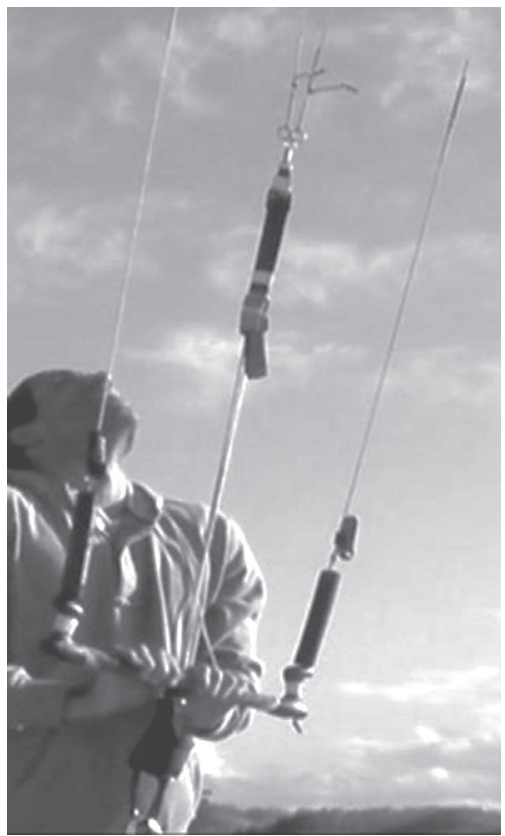

Figura 4. Teste I. (A) Situação em que a barra de controle está na posição elevada. (B) Situação em que a barra de controle está na posição abaixada. 
(A)

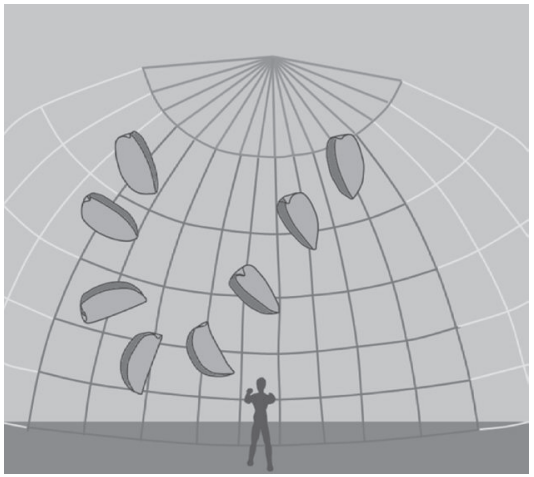

(B)

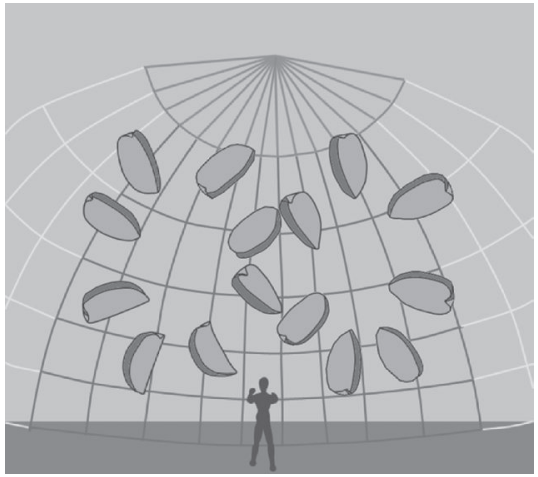

Figura 5. Teste 2. (A) Primeiro mergulho do kite na janela de vento, a qual está representada pelas linhas ao fundo. Dessas linhas, aquelas de cor cinza escuro representam a zona de força e aquelas brancas e de cor cinza claro representam a zona neutra. (B) Segundo mergulho que completa um oito.

A posição do kite compreende o deslocamento vertical e lateral do kite. $\bigcirc$ deslocamento vertical é representado pelo o ângulo vertical (em graus) formado entre as linhas do kite e o plano horizontal (paralelo ao solo), o qual foi representado pela linha tracejada na Figura 6. Esse ângulo foi considerado igual a $0^{\circ}$ quando o vetor da linha do kite estava paralelo ao solo e foi considerado igual a $90^{\circ}$ quando o vetor das linhas do kite encontrava-se perpendicular ao solo.

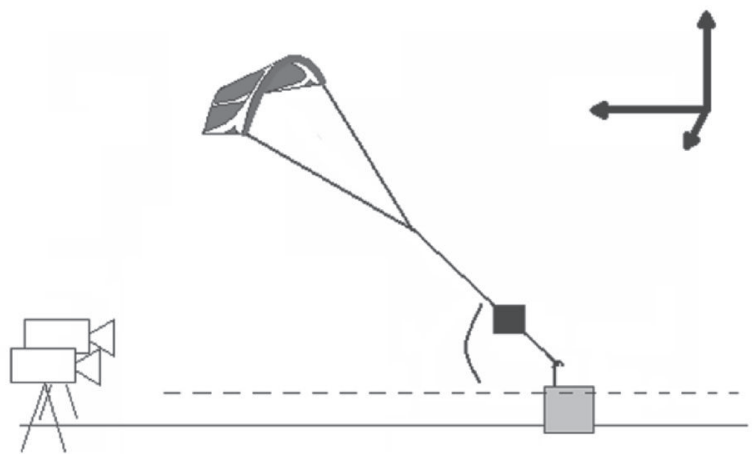

Figura 6. Ângulo vertical. Modelo esquemático - vista lateral - do ambiente de coleta com os eixos do sistema de referências: eixo $Y$ ínfero-superior, o eixo $X$ ântero-posterior e o eixo $Z$ médio-lateral. $O$ ângulo representa o ângulo vertical formado entre as linhas do kite e o plano horizontal, representado pela linha tracejada.

Já o deslocamento lateral é representado pelo ângulo horizontal (em graus) formado entre as linhas do kite e um plano vertical (perpendicular ao solo e paralelo 
ao eixo $X$ do sistema de referência global) que passa aproximadamente entre as câmeras e está representado pela linha tracejada na Figura 7. O ângulo horizontal foi considerado igual a $0^{\circ}$ quando o vetor da linha do kite estava paralelo ao plano perpendicular ao solo. Os valores negativos do ângulo horizontal representaram o lado esquerdo da janela de vento, enquanto que valores positivos representaram o lado direito da janela de vento. Tendo em vista o caráter indicador do sinal (direita ou esquerda), para realização dos cálculos da média e desvio-padrão, foi utilizado o valor absoluto do ângulo horizontal, uma vez que o fator que afeta a força do kite é o ângulo com o plano perpendicular ao solo, independente do lado ser o direito ou o esquerdo. Dessa forma, o centro da janela de vento foi representado pela combinação do ângulo vertical $0^{\circ}$ com o ângulo horizontal $0^{\circ}$.

Logo, o posicionamento do kite é expresso pela combinação desses dois ângulos. Os planos criados para o cálculo dos ângulos foram definidos a partir do sistema de referência global (calibrador). Já o vetor da linha do kite foi definido a partir dos dois marcadores reflexivos posicionados na linha do mesmo (marcadores A e B conforme a Figura 2).

Também foi calculada a distância percorrida pela barra de controle. Para isso, foi determinado o ponto médio entre os marcadores da barra de controle e foi calculada a distância desse ponto até o marcador reflexivo posicionado sobre a linha, o qual estava mais próximo à barra de controle (marcador $\mathrm{B}$ conforme a Figura 2). Dessa distância, foi subtraída a distância mínima entre o ponto médio da barra e o marcador da linha para que eles representassem a distância percorrida pela barra de controle. Assim, quando o valor da distância percorrida foi igual a zero, a barra de controle encontrava-se na posição mais elevada e, o aumento da distância percorrida pela barra significava que esta estava sendo abaixada.
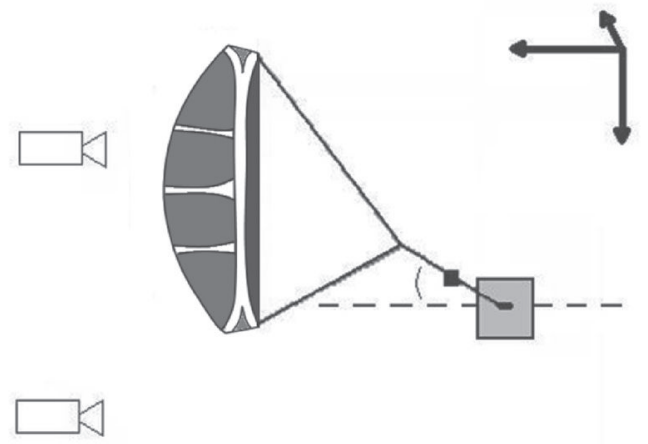

Figura 7. Ângulo horizontal. Modelo esquemático, vista superior do ambiente de coleta com os eixos do sistema de referências: eixo $Y$ ínfero-superior, o eixo $X$ ântero-posterior e o eixo $Z$ médio-lateral. $O$ ângulo representa o ângulo horizontal formado entre as linhas do kite e o plano vertical, representado pela linha tracejada. 
Em relação aos dados de força, eles foram primeiramente filtrados com um filtro passa baixa de ordem três, e a frequência de corte foi determinada por meio da Análise Residual proposta por Winter (2005). Após, formam removidos os valores da força quando a distância percorrida pela barra ultrapassava $0,5 \mathrm{~m}$, já que no teste de abaixar e levantar a barra de controle, a barra foi abaixada até pressionar o chicken loop, o que tirou a tensão da célula de carga.

Além disso, para a análise dos dados de força do teste movimentando o kite na janela de vento em forma de oito, foram selecionados dois pontos de cada mergulho (Figura5): o ponto que apresentou valor máximo e o ponto que apresentou o valor mínimo de força de cada mergulho do kite. Assim, como foram realizados cinco movimentos de oito e cada oito compreende dois mergulhos na janela de vento, foram selecionados dez valores máximos de força e dez valores mínimos de força. Dessa forma, foi possível verificar o posicionamento do kite na janela de vento (combinação dos ângulos horizontal e vertical) no instante em que esses valores ocorreram.

Ainda, a acurácia da coleta dos dados cinemáticos foi determinada de acordo com Barros et al. (2006). O valor da acurácia encontrado para o teste de abaixar e levantar a barra de controle foi igual a $8,9 \mathrm{~mm}$ e, para o teste de movimento do kite na janela de vento foi igual a $9,7 \mathrm{~mm}$. Em relação à dinamometria, pesos conhecidos foram aplicados sob forma de tração na célula de carga. Os valores medidos e os reais foram plotados, e a linearidade da curva foi estabelecida por meio do coeficiente de determinação $\left(R^{2}\right)$, o qual foi igual a 0,9996.

\section{RESULTADOS}

Considerando o teste de abaixar e levantar a barra de controle, os valores de força variaram entre 2,5 e $32 \mathrm{~kg}$. Ainda, para ilustrar o comportamento da força do kite em relação à distância percorrida pela barra de controle, foram escolhidas aleatoriamente uma repetição, a qual foi dividida em uma fase de subida e outra fase de descida. A fase de subida (Figura 8) deve ser observada da direita para a esquerda e, a fase de descida (Figura 8) dever ser observada da esquerda para a direita, já que a distância percorrida pela barra igual a zero corresponde à posição mais elevada da barra de controle como foi explicado na metodologia.

Nesse teste, também foram calculadas as variações dos ângulos horizontal e vertical durante o deslocamento da barra de controle. As Figuras 9 e 10 ilustram a variação desses ângulos durante todas as repetições desse teste. 


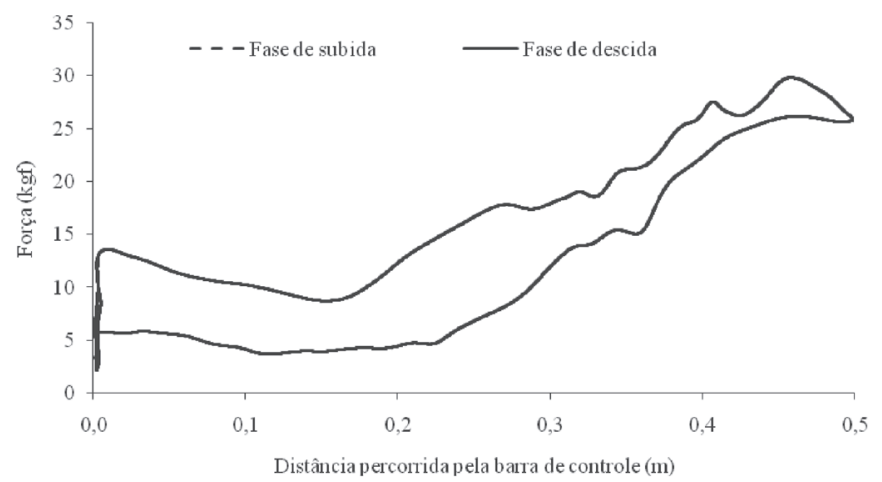

Figura 8. Teste I. Força do kite em relação à distância percorrida pela barra de controle. Como a distância percorrida pela barra de controle igual a zero corresponde à posição mais elevada da barra de controle, a fase de subida deve ser observada da direita para a esquerda e, a fase de descida deve ser observada da direita para a esquerda.

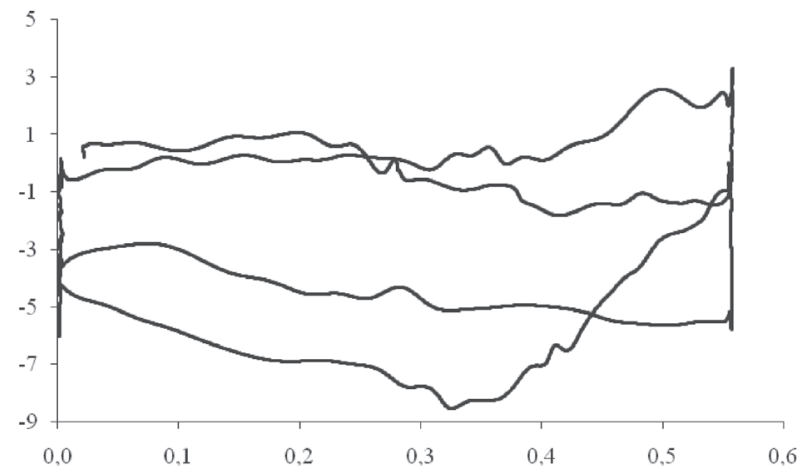

Figura 9. Ângulo horizontal. Variação do ângulo horizontal da linha do kite em relação à distância percorrida pela barra de controle durante todas as repetições do teste de abaixar e levantar a barra de controle.

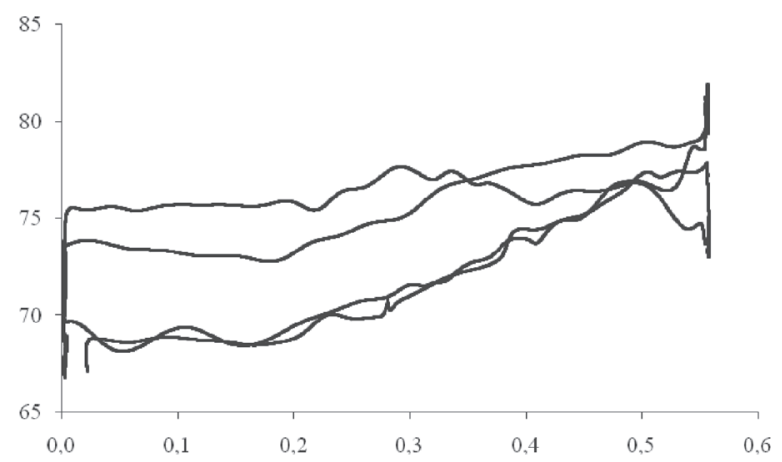

Figura 10. Ângulo Vertical. Variação do ângulo vertical da linha do kite em relação à distância percorrida pela barra de controle durante todas as repetições do teste de abaixar e levantar a barra de controle. 
Considerando o teste de movimentar o kite na janela de vento, os valores máximo e mínimo de força encontrados foram I 10,7 e 2,4 kgf. A média e desviopadrão dos pontos selecionados em cada mergulho do kite, ou seja, dos valores máximos e mínimos, bem como os ângulos vertical e horizontal em que esses valores ocorreram (lembrando que a combinação dos ângulos vertical e horizontal determina a posição do kite) foram apresentados na Tabela I.

Tabela I. Média e desvio-padrão dos valores máximos e mínimos de força encontrados e de seus respectivos ângulos no teste de movimentar o kite na janela de vento.

\begin{tabular}{lll}
\hline & Média & Desvio-padrão \\
\hline Força Máxima (kgf) & 83,1 & 17,6 \\
Ângulo horizontal (graus) & 20,0 & 8,0 \\
Ângulo vertical (graus) & 58,9 & 6,6 \\
& & \\
Força Mínima (kg) & 16,5 & 6,8 \\
Ângulo horizontal (graus) & 19,3 & 5,8 \\
Ângulo vertical (graus) & 73,3 & 6,1 \\
\hline
\end{tabular}

\section{DISCUSSÃO}

Considerando o teste de abaixar e levantar a barra de controle, em todas as repetições realizadas, os valores de força apresentaram um comportamento padrão, aumentando à medida que a barra de controle foi abaixada e diminuindo à medida que a barra de controle foi levantada. Esse resultado vai ao encontro da primeira hipótese do presente estudo.

Além disso, os valores de força encontrados nesse teste foram semelhantes ao relato de Paelinck (20।0), o qual aparentemente avaliou dois kites feitos de materiais diferentes e com área de $8 \mathrm{~m}^{2}$. Nesse relato, foram encontrados valores máximos de aproximadamente $25 \mathrm{~kg}$, enquanto que, no presente estudo, os valores máximos foram em torno de $32 \mathrm{~kg}$. No entanto, deve-se considerar que, no estudo de Paelinck (2010), a velocidade do vento foi de $5 \mathrm{~m} / \mathrm{s}$, enquanto que a velocidade do vento variou entre $9,8 \mathrm{~m} / \mathrm{s}$ e II,3 m/s no presente estudo. Também deve-se considerar a diferença do tamanho e do modelo dos kites utilizados, porque tanto esses fatores citados quanto a velocidade do vento afetam os valores de força do kite. Dessa forma, a diferença encontrada entre o relato e o presente estudo é aceitável, visto que uma maior velocidade de vento combinada com um 
kite de maior área implica em uma maior força de acordo com a teoria da mecânica dos fluidos (BERGER; GROOT; HOLLANDER, 1995; LAUDER; DABNICHKI; BARTLETT, 200I).

Ainda nesse teste, os valores de força apresentaram uma oscilação de 2,5 a 15,0 kgf quando a barra de controle estava levantada (Figuras 7 e 8 quando a distância percorrida é próxima de zero). As possíveis explicações para isso são: primeiro, que essas variações sejam causadas pela variação da velocidade do vento. E, segundo, pelo fato de que nessa situação as linhas presas ao bordo de fuga estavam com uma menor tensão, deixando o kite livre para realizar pequenas mudanças do ângulo de ataque.

Sobre a variação dos ângulos vertical e horizontal no teste de abaixar e levantar a barra de controle, as variações do ângulo horizontal (Figura 9) foram, provavelmente, provocadas por pequenas assimetrias das extremidades da barra de controle durante os movimentos de abaixar e levantar. Dessa forma, parece não haver uma tendência definida no ângulo horizontal (aumento ou diminuição) quando a barra se desloca, de forma que se obtém uma variação aleatória desse ângulo com o deslocamento para cima e para baixo da barra.

Já o ângulo vertical (Figura I0) apresentou uma tendência de um leve aumento com o deslocamento da barra de controle para baixo. Isso, provavelmente, se deve ao aumento da força de sustentação, pois, abaixar a barra de controle faz com que ângulo de ataque aumente (BLOUIN JUNIOR; ISABELLA; RODDEN, 2007), provocando o aumento da força de sustentação. Isso, consequentemente, fez com que o kite tencionasse mais intensamente as linhas e se movimentasse na direção em que seu bordo de ataque está apontado. Logo, quando a barra de controle é abaixada, além de aumentar os valores de força do kite, essa ação também tem influência sobre o posicionamento (movimento) do kite.

Sobre o teste de movimento do kite na janela de vento, tanto os valores máximos quanto os mínimos de força ocorreram próximos ao ângulo horizontal de $20^{\circ}$ (Tabela I). Dessa forma, parece que o ângulo vertical exerce maior influência sobre os valores de força. Além disso, os valores de força aumentaram à medida que o kite se posicionou na zona de força da janela de vento, pois as médias dos ângulos verticais foram menores para os valores máximos de força e o ângulo de $20^{\circ}$ do ângulo horizontal pode ser considerado como próximo do centro da janela de vento.

É importante destacar que, a partir de Blouin Junior, Isabella, Rodden (2007) e de acordo com a posição angular do kite definida pelo presente estudo, a zona de força da janela de vento compreende a combinação dos menores ângulos verticais com os ângulos horizontais mais próximos de zero. E, consequentemente, a zona neutra da janela de vento compreende as combinações de ângulos em que pelo 
menos um dos ângulos não corresponda à descrição da zona de força, ou seja, para que o kite esteja na zona neutra o ângulo vertical deve ser próximo de $90^{\circ}$ e/ou o ângulo horizontal deve ser distante de zero.

Dessa forma, a partir dos resultados do teste de movimentar o kite na janela de vento (Tabela I), foi possível melhor definir a zona de força e a zona neutra da janela de vento. A zona de força compreendeu os pontos em que as linhas do kite apresentaram, aproximadamente, uma combinação de um ângulo vertical inferior a $60^{\circ} \mathrm{com}$ um ângulo horizontal menor que $20^{\circ}$. Enquanto que zona neutra compreendeu, aproximadamente, as combinações de ângulos em que o ângulo vertical foi maior do que $60^{\circ}$ e/ou o ângulo horizontal foi maior do que $20^{\circ}$.

Além disso, nesse teste, os valores de força máximo e mínimo encontrados ( 1 1 0,7 e 2,4 kgf) foram semelhantes aos encontrados no estudo realizado por Walls e Gale (200 I). Nesse estudo citado, foi avaliada a força aplicada no trapézio (equipamento também utilizado no Kitesurfing) na prática de windsurfing durante o velejo com velocidade de vento entre 6, 17 e 15,43 m/s. Foram utilizadas duas diferentes velas, uma de 6,5 $\mathrm{m}^{2}$ (utilizada em situações de vento mais fraco) e outra de 5,0 $\mathrm{m}^{2}$ (utilizada em situação vento mais forte). Os resultados apresentaram valor mínimo de força igual a 0 kgf, e valor máximo de força igual a I I I,5 kgf na utilização da vela com área de 5,0 $\mathrm{m}^{2}$ com velocidade média de vento de $12,86 \mathrm{~m} / \mathrm{s}$. E, valor mínimo de força igual a 0 kgf, e valor máximo de força igual a I 31 , I kgf na utilização da vela com área de $6,5 \mathrm{~m}^{2}$ com velocidade média de vento de $7,2 \mathrm{~m} / \mathrm{s}$.

Ainda, no teste movimentando do kite na janela de vento, também foi analisado o deslocamento da barra de controle, porque o posicionamento da barra poderia afetar os valores de força. Nessa análise, foi constatado que para realizar os oitos, o indivíduo movimentou a barra de controle entre 0 e 0,25 m, ou seja, ele sempre manteve a barra de controle da metade para a posição mais elevada, nunca a abaixando completamente. Assim, não parece haver uma relação entre o deslocamento da barra de controle e os valores de força.

De um modo geral, visto que existe uma carência de pesquisas científicas em relação ao kitesurfing, mais especificamente na quantificação das forças envolvidas, esse estudo compreendeu um primeiro passo na avaliação dessas forças. Ainda, a metodologia descrita poderia ser utilizada como meio de comparar diferentes equipamentos de kite (diferentes tamanhos, modelos, marcas). Outra implicação compreende na melhora da definição da zona de força da janela de vento, a qual pode ser utilizada por instrutores na iniciação da prática do kitesurfing, acelerando o aprendizado do esporte e evitando acidentes comuns na fase de aquisição do domínio do controle do kite. 
É evidente que a presente investigação apresenta limitações. Uma delas compreende o não controlamento constante da velocidade do vento durante todos os testes, visto que a velocidade do vento pode apresentar variações momentâneas bruscas. Outras limitações foram a não verificação direta do ângulo de ataque e da velocidade do kite em relação ao vento durante o teste de movimentar o kite na janela de vento, visto que a área perpendicular do fólio (kite) ao fluido e a velocidade do mesmo em relação ao fluido, no qual ele se desloca, tem influência sobre as forças dinâmicas, força de sustentação e arrasto (LAUDER; DABNICHKI; BARTLETT, 200 I). Outro ponto que limita esse trabalho compreende a análise do comportamento da força a partir dos testes realizados por somente um praticante de kitesurfing, porque, talvez, exista uma diferença no padrão de movimento entre praticantes, o que poderia alterar a força do kite.

Dessa forma, novos estudos que verifiquem o comportamento da força do kite durante o deslocamento do mesmo dentro da janela de vento, considerando a velocidade de deslocamento do kite em relação ao ar são necessários. Entre esses estudos é interessante que sejam avaliados mais indivíduos e outros movimentos (além do oito), por exemplo, o movimento de kite looping, o qual é bastante realizado na prática e empiricamente sabe-se que produz um nível elevado de força, permitindo ( I ) uma comparação entre a força gerada pelo kite entre os movimentos e (2) uma análise de diferentes padrões de movimento entre atletas. Além disso, a medição da força do kite na situação prática do esporte com a verificação do posicionamento do kite (ângulos das linhas) e da barra de controle é com certeza um grande desafio.

\section{CONCLUSÃO}

A partir dos resultados encontrados, foi possível analisar o comportamento da força do kite em relação ao deslocamento da barra de controle e ao posicionamento do kite dentro da janela de vento. Foi observado que os valores de força variaram entre 2,5 e 32 kgf no teste de abaixar e levantar a barra de controle. Ainda, os valores de força aumentaram à medida que a barra de controle foi abaixada e diminuíram à medida que a barra de controle foi levantada. Além disso, abaixar a barra de controle também influenciou o posicionamento do kite, aumentando o ângulo vertical, ou seja, movimentando-o na direção do seu bordo de ataque.

Sobre os valores de força com o kite sendo movimentado dentro da janela de vento, foram encontrados valores entre I I 0,7 e 2,4 kgf. Os resultados sugerem que o ângulo vertical exerceu maior influência sobre a força. $E$, que os maiores valores de força aconteceram nas combinações de ângulos verticais menores que $60^{\circ}$ com ângulos horizontais menores que $20^{\circ}$. 
A partir desses resultados encontrados, a aplicação prática do presente estudo compreende a delimitação da janela de vento. Assim, a zona de força da janela de vento consiste na combinação de um ângulo vertical entre $0^{\circ}$ e $60^{\circ} \mathrm{com}$ um ângulo horizontal entre $0^{\circ}$ e 20. Já a zona neutra corresponde à combinação de um ângulo vertical entre $60^{\circ}$ e $90^{\circ} \mathrm{com}$ qualquer ângulo horizontal. Isso pode auxiliar os instrutores na introdução desse conceito para iniciantes.

Considerando o teste de abaixar e levantar a barra de controle, pode-se presumir que ao combinar o movimento de abaixar a barra de controle com o posicionamento do kite em um ponto da zona de força da janela de vento, serão alcançados valores altos de força. Na prática do esporte, a combinação desses dois recursos permite ao praticante velejar mais rápido e realizar saltos.

Esses achados constituem o primeiro passo para compreender o comportamento das forças do kite. No entanto, são necessários mais dados científicos, como: (I) a realização desses testes no mesmo kite em outras condições de vento, (2) teste de outros modelos e tamanhos de kites, (3) teste de kites realizando o kite looping, (4) análise de padrões de movimento e, provavelmente o mais importante, (5) testes da força do kite em uma situação real de velejo.

\section{Description of the behavior of the kite's force in relation to its position and the displacement of the control bar}

ABSTRACT: Introduction. During kitesurfing, the sailors propel themselves changing the kite's attack angle in two ways: moving the kite in the wind window and moving the control bar. Objective. To describe the behavior of the kite's forces in relation to its position in the wind window and the distance traveled by the control bar. Methods. Videogrammetry and dynamometry were used. Two tests were performed: moving the control bar and moving the kite in the wind window. Results. The force increased while the bar was moved down. Moving the kite, the power zone was defined as the combination of vertical angles smaller than $60^{\circ}$ with horizontal angles smaller than $20^{\circ}$. Conclusion. The force increases when the bar goes down. The vertical angle seems to be more important to the force values.

KEYWORDS: Extreme sports; nautical sports; wind window; attack angle.

Descripción del comportamiento de la fuerza del kite en relación a su posición y a el desplazamiento de la barra de control

RESUMEN: Introducción. Nel kitesurfing, lo deportista navega cambiando el ángulo de ataque del kite de dos formas: moviendo el kite en la ventana de viento y moviendo la barra de control. Objetivo. Describir el comportamiento de la fuerza del kite en relación a su posicionamiento en la ventana de viento y al desplazamiento de la barra de control. Métodos. Fueron utilizados sistemas de videogrametría y dinamometría, en dos situaciones: moviendo el kite y moviendo la barra. Resultados. La fuerza aumento cuando la barra fue bajada. Moviendo el kite, la 
área de fuerza fue definida por la combinación de ángulos verticales menores que $60^{\circ} \mathrm{con}$ ángulos horizontales menores que $20^{\circ}$. Conclusión. La fuerza aumenta cuando la barra baja. El ángulo vertical puede ejercer más influencia en la fuerza.

PALABRAS CLAVE: Deportes extremos; deportes acuáticos; ventana de viento; angulo de ataque.

\section{REFERÊNCIAS}

BARROS, R. M. L., et al. Desenvolvimento e avaliação de um sistema para análise tridimensional de movimentos humanos. Revista Brasileira de Engenharia Biomédica, Campinas, v. 15, n. I-2, p. 79-86, jan./ago., 1999.

BARROS, R. M. L., et al. A method to synchronize video cameras using the audio band. Journal of Biomechanics, Champaign, v. 39, n. 4, p. 776-780, dez., 2006.

BERGER, M.; GROOT, G.; HOLLANDER, P. Hydrodynamic drag and lift forces on human hand/arm models. Journal of Biomechanics, Champaign, v. 28, n. 2, p. I25-133, abr, 1995.

BLOUIN JUNIOR, M. R.; ISABELLA, B. E.; RODDEN, J. E. Wind power from kites. A Major Qualifying Project. Faculty of the Worcester Polytechnic Institute. Worcester, 2007.

COSTA, A. V. O potencial das atividades físicas de aventura na natureza em Porto Alegre: um estudo integrado dos critérios de adequação do local, da infra-estrutura, das contribuições sócio-ambientais e dos riscos. 2006. 228 f. Dissertação (Mestrado) - Programa de Pósgraduação em Ciência do Movimento Humano, Universidade Federal do Rio Grande do Sul, Porto Alegre, 2006.

LAUDER, M. A.; DABNICHKI, P.; BARTLETT, R. M. Improved accuracy and reliability of sweepback angle, pitch angle and hand velocity calculations in swimming. Journal of Biomechanics, Champaign, v. 34, n. I, p.31-39, jul./dez. 200 I.

LOSS, J. F., et al. Sincronismo entre Cinemetria e Eletromiografia. In: CONGRESSO BRASILEIRO DE BIOMECÂNICA, 7., 1997, Campinas. Anais... Campinas, 1997. p. 453-456.

LUNDGREN, L., et al. Biomechanics of extreme sports - a kite surfing scenario. In: ANNUAL CONGRESS OF THE NORDIC ERGONOMICS SOCIETY, 39, 2007, Buhuslän Proceedings...Buhuslän: Abstract book, 2007. p. 169. Disponível em: <http://www.nordiskergonomi.org/nes2007/CD_NES_2007/papers/Abstract_NES2007.pdf> Acesso em: 20 maio de 2010.

NICKEL, C. et al. A Prospective Study of Kitesurfing Injuries. American Journal of Sports Medicine, Baltimore, v. 32, n.4, p. 921-927, jun. 2004.

PAELINCK, R. Is a Kite's Weight Important? Disponível em: <http://www.thekiteboarder. com/wordpress/20 I0/04/is-a-kites-weight-important/> Acesso em: I maio 2010. 
SANDERS, R. Hydrodynamic Characteristics of a Swimmer's Hand. Journal of Applied Biomechanics, Champaign, v. I5, n. p. 3-27, fev., 1999.

SPANJERSBERG, W. R.; SCHIPPER, I. B.. Kitesurfing: When Fun Turns to Trauma-The Dangers of a new extreme sport. The Journal of Trauma, Baltimore, v. 63, n.3 . p. E76-80, set. 2007.

VERCRUYSSEN, F. et al. Assessment of physiological demand in kitesurfing. European Journal of Applied Physiology, Berlin, v. 105, n. I, p. 103-109, jan. 2009.

WALLS, J. t.; GALE, T. j. A Technique for the Assessment of Sailboard Harness line force. Journal of Science and Medicine in Sport, Belconnen, v. 4, n. 3, p. 348-356, set. 2001.

WINTER, D. Kinematics. In: WINTER, D. Biomechanics and Motor Control of Human Movement. New Jersey: John Wiley \& Sons, 2005. p. 13-58.

Recebido: 28 jul. 2010 Aprovado: 03 maio 2011

Endereço para correspondência: Victor Wigner Tremea Rua Dona Oti, 208, apto. 402 Bairro Petrópolis Porto Alegre - RS CEP: 90680-060 